\title{
On the state of low luminous accreting neutron stars
}

\author{
N. R. Ikhsanov ${ }^{1,2, \star}$ \\ 1 Max-Planck-Institut für Radioastronomie, Auf dem Hügel 69, 53121 Bonn, Germany \\ 2 Central Astronomical Observatory of the Russian Academy of Science at Pulkovo, Pulkovo 65-1, \\ 196140 Saint-Petersburg, Russia
}

Received 5 March 2001 / Accepted 14 June 2001

\begin{abstract}
Observational appearance of a neutron star in the subsonic propeller state which is a companion of a wind-fed mass-exchange close binary system is discussed. During the subsonic propeller state (which was first introduced by Davies et al. 1979) the neutron star magnetosphere is surrounded by a spherical quasi-static plasma envelope, which is extended from the magnetospheric boundary up to the star accretion radius. The energy input to the envelope due to the propeller action by the neutron star exceeds the radiative losses and the plasma temperature in the envelope is of the order of the free-fall temperature. Under this condition the magnetospheric boundary is interchange stable. Nevertheless, I find that the rate of plasma penetration from the envelope into the magnetic field of the neutron star due to diffusion and magnetic field line reconnection processes is large enough for the accretion power to dominate the spindown power. I show that the accretion luminosity of the neutron star in the subsonic propeller state is $L_{\mathrm{a}} \simeq 5 \times 10^{30} \div 10^{33} M_{15} \mathrm{erg} \mathrm{s}^{-1}$, where $\dot{M}_{15}$ is the strength of the normal companion stellar wind which is parametrized in terms of the maximum possible mass accretion rate onto the neutron star magnetosphere. On this basis I suggest that neutron stars in the subsonic propeller state are expected to be observed as low luminous accretion-powered pulsars. The magnetospheric radius of the neutron star in this state is determined by the strength of the stellar wind, $\dot{M}_{\mathrm{c}}$, while the accretion luminosity is determined by the rate of plasma penetration into the star magnetosphere, $\dot{M}_{\mathrm{a}}$, which is $\dot{M}_{\mathrm{a}} \ll \dot{M}_{\mathrm{c}}$. That is why the classification of the neutron star state in these objects using the steady accretion model (i.e. setting $\dot{M}_{\mathrm{a}}=\dot{M}_{\mathrm{c}}$ ) can lead to a mistaken conclusion.
\end{abstract}

Key words. accretion - magnetic fields - stars: close: binaries - neutron stars

\section{Introduction}

As was first recognized by Shvartsman (1970), three states of a rotating, magnetized neutron star in a close binary system can be distinguished: ejector, propeller and accretor. This classification reflects three different evolutionary stages and three different mechanisms of energy release responsible for the neutron star emission. The sequence of states which a magnetized neutron star in a wind-fed mass-exchange binary system follows as it spins down from the initially very short periods can be expressed in the form of the following chain: ejector $\rightarrow$ propeller $\rightarrow$ accretor.

Neutron stars in the state of ejector are known as spinpowered pulsars, in which the spindown power,

$L_{\mathrm{md}}=\frac{2 \mu^{2} \sin ^{2} \beta}{3 c^{3}} \omega^{4}$,

dominates the star energy budget and is spent predominantly on the generation of magneto-dipole waves and particle acceleration. Here $\mu$ is the neutron star magnetic

* e-mail: ikhsanov@mpifr-bonn.mpg.de moment, $\beta$ is the angle between the rotational and magnetic axes, $\omega=2 \pi / P_{\mathrm{s}}$ is the star angular velocity, $P_{\mathrm{s}}$ is the neutron star spin period and $c$ is the speed of light.

The state of a neutron star is classified as accretor if the star luminosity is dominated by the accretion power,

$L_{\mathrm{a}}=\dot{M}_{\mathrm{a}} \frac{G M_{\mathrm{ns}}}{R_{\mathrm{ns}}}$.

Here $M_{\mathrm{ns}}$ and $R_{\mathrm{ns}}$ are the mass and the radius of the neutron star, respectively, and $\dot{M}_{\mathrm{a}}$ is the mass accretion rate onto the neutron star surface.

The intermediate state is called propeller. The necessity to introduce this state can be justified as follows. The pulsar-like spindown ceases when the pressure of the material being ejected by the neutron star,

$p_{\text {out }}=\frac{L_{\mathrm{md}}}{4 \pi c R_{\alpha}^{2}}$,

can no longer balance the pressure of the surrounding gas,

$p_{\infty}=\frac{1}{2} \rho_{\infty} V_{\text {rel }}^{2}$.

Here $\rho_{\infty}$ is the density of the surrounding gas, $V_{\text {rel }}$ is the relative velocity between the neutron star and the 
surrounding gas, and $R_{\alpha}$ is the accretion radius of the neutron star,

$R_{\alpha}=\frac{2 G M_{\mathrm{ns}}}{V_{\mathrm{rel}}^{2}}$.

Combining Eqs. (3) and (4) one finds that this occurs when the neutron star spin period $P_{\mathrm{s}}=P_{\mathrm{md}}$, where

$P_{\mathrm{md}}=0.3 \mu_{30}^{1 / 2} V_{8}^{-1 / 4}(\sin \beta)^{1 / 2}\left[\frac{\dot{M}_{\mathrm{c}}}{10^{15} \mathrm{~g} \mathrm{~s}^{-1}}\right]^{-1 / 4} \mathrm{~s}$.

Here $\mu_{30}=\mu / 10^{30} \mathrm{G} \mathrm{cm}^{3}, V_{8}=V_{\text {rel }} / 10^{8} \mathrm{~cm} \mathrm{~s}^{-1}$ and $\dot{M}_{\mathrm{c}}$ is the strength of the normal companion stellar wind which is parametrized in terms of the maximum possible accretion rate following ${ }^{1}$ Davies et al. (1979)

$\dot{M}_{\mathrm{c}}=\pi R_{\alpha}^{2} \rho_{\infty} V_{\mathrm{rel}}$.

On the other hand, for the steady accretion onto the neutron star surface to realize the canonical magnetospheric radius, which is defined by equating the ram pressure of the inflowing material with the magnetic pressure due to the dipole field of the neutron star,

$R_{\mathrm{m}} \equiv\left(\frac{\mu^{2}}{\dot{M}_{\mathrm{c}} \sqrt{2 G M_{\mathrm{ns}}}}\right)^{2 / 7}$,

should be smaller than the corotational radius ${ }^{2}$,

$R_{\mathrm{cor}}=\left(\frac{G M_{\mathrm{ns}}}{\omega^{2}}\right)^{1 / 3}$.

Otherwise the neutron star proves to be in the centrifugal inhibition regime (i.e. the centrifugal acceleration at the magnetospheric boundary, $\omega^{2} R_{\mathrm{m}}$, dominates the gravitational acceleration, $\left.G M_{\mathrm{ns}} / R_{\mathrm{m}}^{2}\right)$ and hence, the plasma accretion onto the star surface is impossible. Putting Eqs. (6) to (9) one finds that the condition $r_{\mathrm{m}} \lesssim R_{\text {cor }}$ is satisfied only if $\dot{M}_{\mathrm{c}} \gtrsim \dot{M}_{\text {ea }}$, where

$\dot{M}_{\text {ea }} \simeq 0.75 \mu_{30}^{2} V_{8}^{7 / 5} m^{4}(\sin \beta)^{-14 / 5} M_{\odot} \mathrm{yr}^{-1}$.

Here $m$ is the mass of the neutron star expressed in units of $M_{\odot}$. However so huge a mass transfer rate can never be realized in a wind-fed mass-exchange close binary system $\left(\dot{M}_{\text {ea }}\right.$ exceeds the maximum possible rate of mass loss by O-type supergiants by more than five orders of magnitude!). That is why the direct ejector $\rightarrow$ accretor state

1 There should be no confusion between $\dot{M}_{\mathrm{c}}$, which is the mass of the surrounding material interacting with the neutron star moving through the wind in a time unit and $\dot{M}_{\mathrm{a}}$, which is the mass accretion rate onto its surface. In the general case $\dot{M}_{\mathrm{c}} \neq \dot{M}_{\mathrm{a}}$. The case of $\dot{M}_{\mathrm{c}}=\dot{M}_{\mathrm{a}}$ corresponds to the steady accretion process, when all material captured by the neutron star reaches the star surface. In this particular case $\dot{M}_{\mathrm{c}}$ is called the rate of mass capture by the neutron star (Bondi \& Hoyle 1944). However, as it will be shown below, it has a different physical meaning if the neutron star is in the state of propeller.

2 This is a necessary but not a sufficient condition. transition under the conditions of interest cannot be realized and the evolutionary track of the initially fast rotating magnetized neutron star must contain an additional spindown state which is traditionally called propeller.

A neutron star in the state of propeller is spinning down due to the interaction between its fast rotating magnetosphere and the surrounding material. The mechanism of this interaction has been investigated in detail by Davies et al. (1979) and Davies \& Pringle (1981) who have shown that during the propeller state the star magnetosphere is surrounded by a spherical quasi-static envelope in which the plasma temperature is of the order of the free-fall temperature,

$T(R) \simeq T_{\mathrm{ff}}(R)=\frac{G M_{\mathrm{ns}} m_{\mathrm{p}}}{k R}$

Here $m_{\mathrm{p}}$ is the proton mass and $k$ is the Boltzmann constant. The rotational energy loss by the neutron star is convected up through the envelope by the turbulent motions and lost through its outer boundary. The structure of the envelope and the spindown rate of the star depend on the value of the ratio:

$\kappa=\frac{\omega R_{\mathrm{m}}}{V_{\mathrm{s}}\left(R_{\mathrm{m}}\right)}$

where $R_{\mathrm{m}}$ is the magnetospheric radius of the neutron star and $V_{\mathrm{s}}\left(R_{\mathrm{m}}\right)$ is the sound speed in the envelope plasma, which according to Eq. (11) is of the order of the free-fall velocity, $V_{\mathrm{ff}}$ :

$V_{\mathrm{s}}\left(R_{\mathrm{m}}\right) \simeq V_{\mathrm{ff}}\left(R_{\mathrm{m}}\right)=\sqrt{\frac{2 G M_{\mathrm{ns}}}{R_{\mathrm{m}}}}$.

On this basis, Davies et al. (1979) distinguished three sub-states of the propeller state ${ }^{3}$ : (b) very rapid rotator $(\kappa \gg 1)$; (c) supersonic propeller $(\kappa \gtrsim 1)$ and $(\mathrm{d})$ subsonic propeller $(\kappa<1)$.

In cases "b" and "c" the magnetospheric radius of the neutron star exceeds its corotational radius. The neutron star is in the centrifugal inhibition regime and no accretion onto its surface occurs. The spindown power dominates the energy budget and is comparable to the radiative losses of the envelope.

When the neutron star spin period decreases below the critical period,

$P_{\mathrm{cd}}=23 \mu_{30}^{6 / 7} m^{-5 / 7}\left[\frac{\dot{M}_{\mathrm{c}}}{10^{15} \mathrm{~g} \mathrm{~s}^{-1}}\right]^{-3 / 7} \mathrm{~s}$,

the corotational radius reaches the magnetospheric radius. Under the condition $P_{\mathrm{s}}>P_{\mathrm{cd}}$ the wind plasma, penetrating from the base of the envelope into the star magnetic field, is able to flow along the magnetic field lines and to accrete onto the star surface. However the rate of plasma penetration into the star magnetic field remains essentially

\footnotetext{
3 According to their classification, case "a" corresponds to the pulsar-like spindown, i.e. the ejector state.
} 
smaller than $\dot{M}_{\mathrm{c}}$ if the magnetospheric boundary is stable with respect to interchange instabilities (e.g. RayleighTaylor instability). According to Arons \& Lea (1976a) and Elsner \& Lamb (1976), the onset condition for the interchange instability of the magnetospheric boundary reads

$T\left(R_{\mathrm{m}}\right)<T_{\mathrm{cr}} \simeq 0.3 T_{\mathrm{ff}}$.

This means that the steady accretion process under the condition $P_{\mathrm{s}}>P_{\mathrm{cd}}$ can be realized only if the cooling of the envelope plasma (due to the radiation and convective motions) dominates the energy input to the envelope due to the propeller action by the neutron star.

Investigating this particular state, Davies \& Pringle (1981, hereafter DP81) have shown that the cooling processes in the envelope are effective if the spin period of the star exceeds a so-called break period $P_{\mathrm{br}}$, which according to Ikhsanov (2001a) is

$P_{\mathrm{br}} \simeq 450 \mu_{30}^{16 / 21} \dot{M}_{15}^{-5 / 7} \mathrm{~m}^{-4 / 21} \mathrm{~s}$.

DP81 have assumed that during the epoch when $P_{\mathrm{cd}}<$ $P_{\mathrm{s}}<P_{\mathrm{br}}$, no accretion onto the star surface is realized (i.e. $\dot{M}_{\mathrm{a}}=0$ ) and the star energy budget is dominated by the spindown power, which in the case of this - subsonic propeller - state is

$L_{\mathrm{ssp}}=6.4 \times 10^{31} \mathrm{erg} \mathrm{s}^{-1} \mu_{30}^{2} \mathrm{~m}^{-1} P_{25}^{-3}$,

where $P_{25}=P_{\mathrm{s}} / 25 \mathrm{~s}$.

At the same time, the assumption $\dot{M}_{\mathrm{a}}=0$ under the condition $R_{\mathrm{m}}<R_{\text {cor }}$ is not obvious since the interchange instability is not the only mode by which the accreting plasma can enter the neutron star magnetic field. In this Paper I show that the accretion luminosity of the neutron star in the subsonic propeller state can be comparable or even exceeds the spindown power if the plasma penetration into the magnetosphere is governed by the magnetic field lines reconnection process (Sect. 2). In this case the star can be observed as a low luminous accretion-powered $\mathrm{X}$-ray pulsar rather than the spin-powered propeller. The results are discussed and summarized in Sect. 3.

\section{Accretion luminosity of a neutron star in "subsonic propeller" state}

I consider a close binary system in which a rotating, magnetized neutron star is orbiting around a high-mass main sequence companion which underfills its Roche lobe and loses mass in the form of stellar wind. The neutron star, which moves through the wind of the normal companion, is assumed to be in the state of subsonic propeller. This means that the neutron star magnetosphere is surrounded by a hot plasma envelope ( $T \approx T_{\mathrm{ff}}$ ) which is extended from the magnetospheric boundary, $R_{\mathrm{m}} \lesssim R_{\text {cor }}$, up to the accretion radius of the neutron star, $R_{\alpha}$. The spin period of the neutron star lies within the interval $P_{\mathrm{cd}}<P_{\mathrm{s}}<P_{\mathrm{br}}$, and thus, the rate of energy input to the envelope due to the propeller action by the neutron star (expressed by Eq. (16)) dominates the radiative losses from the envelope.
Under this condition the envelope can be considered as a static adiabatic atmosphere in which the plasma pressure $p \propto R^{-5 / 2}$, the sound speed $c_{\mathrm{s}} \propto R^{-1 / 2}$ and the number density $n \propto R^{-3 / 2}$. The plasma pressure at the outer edge of the envelope is equal to the ram pressure of the surrounding gas. The formation of the envelope in the first approximation prevents the stellar wind from penetrating into the accretion lobe of the neutron star (i.e. the sphere with the radius $R_{\alpha}$ ). In this case stellar wind overflows the outer edge with the rate $\dot{M}_{\mathrm{c}}$ compressing and heating the envelope plasma (for discussion see DP81).

The equilibrium shape of a neutron star magnetosphere surrounded by a hot hydrogen envelope has been reconstructed by Arons \& Lea (1976a), Elsner \& Lamb (1977) and Michel (1977). All these authors have argued that the magnetosphere in the considered case tends to be closed and its boundary is convex towards the accreting plasma, with two cusp points situated on the magnetic axis of the neutron star. It is rotationally symmetric about the magnetic axis and reflection symmetric about the equatorial plane.

\subsection{The rate of plasma entry into the star magnetosphere}

Formation of the magnetosphere, to a first approximation, prevents the envelope plasma from reaching the neutron star itself. That is why the rate of mass accretion onto the neutron star surface strongly depends on the rate of plasma penetration into the magnetosphere at its boundary. As was mentioned in the introduction, under the condition $P_{\mathrm{s}}<P_{\mathrm{br}}$, the cooling of the envelope plasma is not effective and hence the magnetospheric boundary is interchange stable. Under this condition the modes by which the envelope plasma can enter the star magnetosphere are (i) turbulent diffusion and (ii) reconnection of the magnetic field lines.

\subsubsection{Diffusion}

The conductivity of the envelope plasma can be approximated by that of a fully ionized gas: $\sigma \sim 10^{7} T_{\mathrm{e}}^{3 / 2}$, where $T_{\mathrm{e}}$ is the electron temperature. In our case, the value of conductivity is very high but, nevertheless, finite $(\sigma<\infty)$. That is why a diffusion skin layer (magnetopause) at the magnetospheric boundary is formed. The thickness of the magnetopause in the general case depends on the velocity and the time of plasma diffusion across the magnetic field lines, but in any case it is larger than the Larmor radius of ions. Under the condition $P_{\mathrm{cd}} \gtrsim P_{\mathrm{s}}$, the gravitation force applied to the plasma in the diffusion layer dominates the centrifugal force. Therefore the plasma penetrating the layer flows along the field lines toward the magnetic poles of the neutron star and accretes onto the star surface.

The rate of plasma flow into the magnetopause from the envelope due to diffusion is

$\dot{M}_{\mathrm{d}} \simeq 4 \pi R_{\mathrm{m}}^{2} \rho\left(R_{\mathrm{m}}\right) V_{\text {diff }}$ 
$\rho\left(R_{\mathrm{m}}\right)$ is the plasma density at the base of the envelope just over the magnetosphere boundary. It can be evaluated equating the plasma ram pressure and the magnetic field pressure at the boundary:

$\rho\left(R_{\mathrm{m}}\right)=\frac{\mu^{2}}{4 \pi G M_{\mathrm{ns}} R_{\mathrm{m}}^{5}}$.

$V_{\text {diff }}$ is the diffusion velocity which, according to Ikhsanov \& Pustil'nik (1996), can be expressed in the following form

$V_{\text {diff }}=\sqrt{D_{\text {eff }} / t_{\mathrm{ff}}}$.

Here $D_{\text {eff }}$ is the effective diffusion coefficient and $t_{\mathrm{ff}}=$ $R_{\mathrm{m}}^{3 / 2} / \sqrt{G M_{\mathrm{ns}}}$ is the free-fall time, which is the characteristic time of the plasma flow from the layer to the star surface.

In the general case the value of $D_{\text {eff }}$ is obviously limited by the following relation:

$D_{\text {cr }} \lesssim D_{\text {eff }} \lesssim D_{\mathrm{B}}$,

where $D_{\mathrm{cr}}=c^{2} / 4 \pi \sigma$ and $D_{\mathrm{B}}=\zeta V_{T(i)} r_{h(i)}$ are the coefficients of the classical and Bohm diffusion, respectively, $V_{T(i)}$ and $r_{h(i)}$ are the thermal velocity and Larmor radius of ions, and $\zeta$ is the diffusion efficiency.

The minimum rate of plasma entry into the magnetosphere can be obtained putting $D_{\text {eff }} \sim D_{\text {cl }}$. In this case one finds

$\dot{M}_{\mathrm{cl}} \simeq 10^{6} \mu_{30}^{2 / 7} \mathrm{~m}^{-15 / 14}\left(\frac{\dot{M}_{\mathrm{c}}}{10^{15} \mathrm{~g} \mathrm{~s}^{-1}}\right)^{6 / 7} \mathrm{~g} \mathrm{~s}^{-1}$.

The maximum inflow rate is realized if the diffusion process is governed by drift-dissipative instabilities (i.e. Bohm diffusion)

$D_{\text {eff }} \simeq D_{\mathrm{B}}=\frac{\zeta c k T_{\mathrm{i}}\left(R_{\mathrm{m}}\right)}{16 e B\left(R_{\mathrm{m}}\right)}$,

where $T_{\mathrm{i}}$ is the ion plasma temperature at the base of the envelope. Combining Eqs. (17)-(21) yields

$\dot{M}_{\mathrm{B}} \sim 310^{10} \mathrm{~g} \mathrm{~s}^{-1} \zeta_{0.1}^{1 / 2} \mu_{30}^{-1 / 14} \mathrm{~m}^{1 / 7}\left(\frac{\dot{M}_{\mathrm{c}}}{10^{15} \mathrm{~g} \mathrm{~s}^{-1}}\right)^{11 / 14}$

where $\zeta_{0.1}=\zeta / 0.1$. This normalization of the diffusion efficiency is adopted following the results of experiments on the nuclear fusion (e.g. Hamasaki et al. 1974) and the measurements of the solar wind penetrating the magnetosphere of the Earth (Gosling et al. 1991).

Hence, the total rate of mass flow into the magnetosphere due to the diffusion process is $\dot{M}_{\mathrm{d}}=\dot{M}_{\mathrm{cl}}+\dot{M}_{\mathrm{B}}$.

\subsubsection{Reconnections}

According to Elsner \& Lamb (1984) the rate of plasma entry into the magnetosphere due to reconnection of the magnetic field lines can be expressed as

$\dot{M}_{\mathrm{rec}} \simeq \alpha_{\mathrm{r}} \frac{\mu^{2}}{G M_{\mathrm{ns}} R_{\mathrm{m}}^{3}} \frac{A_{\mathrm{r}}}{4 \pi R_{\mathrm{m}}^{2}} V_{\mathrm{a}}$, where $\alpha_{\mathrm{r}}$ is the efficiency of the reconnection process, $A_{\mathrm{r}}=$ $4 \pi R_{\mathrm{m}} \lambda_{\mathrm{m}}$ is the effective area of the reconnection region and $V_{\mathrm{a}}=B / \sqrt{4 \pi \rho}$ is the Alfvén velocity, which in the boundary layer at the base of the hot envelope is of the order of $V_{\mathrm{ff}}\left(R_{\mathrm{m}}\right)$.

The values of $\alpha_{\mathrm{r}}$ and $A_{\mathrm{r}}$ depend on the current sheet parameters and the magnetic field configuration in the accretion flow. Investigations of reconnection processes in solar flares and in the Earth's magnetopause suggest the average value of $\alpha_{\mathrm{r}} \approx 0.1$ (see Priest \& Forbes 2000 and references therein).

The effective area of the reconnection region depends of the scale of the magnetic field in the accreting plasma over the magnetospheric boundary. It is limited by the initial inhomogeneity of the accretion flow and/or by the fragmentation of plasma over the rotating magnetospheric boundary due to the Kelvin-Helmholtz instability. In the latter case the average scale of plasma vortices of the embedded field is $\lambda_{\mathrm{m}} \sim 0.1 R_{\mathrm{m}}$ (see Arons \& Lea 1976b; Wang \& Robertson 1985). On the other hand, the scale of the plasma inhomogeneity at the base of the envelope can be evaluated by the size of the convective cell (i.e. by the height of the homogeneous atmosphere over the magnetospheric boundary, $\left.h_{\mathrm{g}}\right)$. In our case $\left(T \gtrsim 0.3 T_{\mathrm{ff}}\right)$ one finds $h_{\mathrm{g}} \gtrsim 0.3 R_{\mathrm{m}}$ and, correspondingly, the ratio of the area of a convective cell to the total area of the magnetospheric boundary is $\left(h_{\mathrm{g}} / R_{\mathrm{m}}\right)^{2} \sim 0.1$. In this context the estimate $\lambda_{\mathrm{m}} \sim 0.1 R_{\mathrm{m}}$ seems to be appropriate.

Under these conditions the rate of plasma penetration into the neutron star magnetic field due to the magnetic lines reconnection reads

$\dot{M}_{\text {rec }} \simeq 10^{13} \mathrm{~g} \mathrm{~s}^{-1}\left[\frac{\alpha_{\mathrm{r}}}{0.1}\right]\left[\frac{\lambda_{\mathrm{m}}}{0.1 R_{\mathrm{m}}}\right]\left(\frac{\dot{M}_{\mathrm{c}}}{10^{15} \mathrm{~g} \mathrm{~s}^{-1}}\right)$.

Thus, the rate of plasma penetration into the magnetosphere of the neutron star in the state of subsonic propeller can be expressed as

$\dot{M}_{\mathrm{a}}=\dot{M}_{\mathrm{d}}+\dot{M}_{\mathrm{rec}}$.

\subsection{Accretion luminosity}

Under the condition $R_{\mathrm{m}}<R_{\text {cor }}$ plasma penetrating the magnetic field of the neutron star is accreted onto its surface. This indicates that the observed total luminosity of the neutron star in the state of subsonic propeller is

$L_{\mathrm{bol}}=L_{\mathrm{ssp}}+L_{\mathrm{a}}$.

The lower limit to the accretion luminosity, $L_{\mathrm{a}}$, can be estimated assuming the plasma entry in the stellar magnetic field from the base of the envelope due to diffusion (see Eq. (22)):

$L_{\text {a,diff }} \sim 410^{30} \zeta_{0.1}^{1 / 2} \mu_{30}^{-1 / 14} \mathrm{~m}^{8 / 7} R_{6}^{-1} \dot{M}_{15}^{11 / 14} \mathrm{erg} \mathrm{s}^{-1}$,

where $\dot{M}_{15}$ is the strength of the stellar wind expressed in units of $10^{15} \mathrm{~g} \mathrm{~s}^{-1}$. 
Combining Eqs. (16) and (27) one finds that the ac-

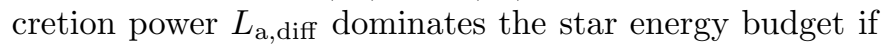
$P_{\mathrm{s}} \gtrsim P_{\mathrm{dda}}$, where

$P_{\text {dda }}=63 \zeta_{0.1}^{1 / 6} \mu_{30}^{29 / 42} m^{-5 / 7} R_{6}^{1 / 3} \dot{M}_{15}^{-11 / 42} \mathrm{~s}$.

If the plasma penetration the magnetosphere of a neutron star is governed by the reconnection of the magnetic field lines, the accretion luminosity of the star is

$L_{\mathrm{a}, \mathrm{rec}} \simeq 10^{33} \alpha_{0.1} \mathrm{mR}_{6}^{-1} \lambda_{0.1} \dot{M}_{15} \mathrm{erg} \mathrm{s}^{-1}$,

where $\alpha_{0.1}=\alpha_{\mathrm{r}} / 0.1$ and $\lambda_{0.1}=\lambda_{\mathrm{m}} / 0.1 R_{\mathrm{m}}$. In this case the accretion power already dominates the spindown power at $P_{\text {cd }}$ if $\dot{M}_{\mathrm{c}}>\dot{M}_{\text {rda }}$, where

$\dot{M}_{\text {rda }} \simeq 610^{13} \mu_{30}^{2} \mathrm{~m}^{-2} R_{6} \alpha_{0.1}^{-1} \lambda_{0.1}^{-1} P_{25}^{-3} \mathrm{~g} \mathrm{~s}^{-1}$.

In both cases the neutron star will be recognized as a low luminous accretion-powered pulsar in which the radius and the temperature of polar caps are ${ }^{4}$

$$
\begin{aligned}
& R_{\mathrm{pc}}=0.3 \mu_{30}^{-2 / 7} m^{1 / 14} R_{6}^{3 / 2}\left[\frac{\dot{M}_{\mathrm{c}}}{10^{15} \mathrm{~g} \mathrm{~s}^{-1}}\right]^{1 / 7} \mathrm{~km}, \\
& T_{\mathrm{pc}} \simeq 8 \times 10^{6}\left[\frac{R_{\mathrm{pc}}}{0.3 \mathrm{~km}}\right]^{-1 / 2}\left[\frac{L_{\mathrm{a}}}{10^{33} \mathrm{erg} \mathrm{s}^{-1}}\right]^{1 / 4} \mathrm{~K} .
\end{aligned}
$$

\subsection{Quasi-static envelope approximation}

The model of the envelope which is surrounding the magnetosphere of a neutron star in the subsonic propeller state has been constructed by DP 81 under the assumption that no accretion onto the star surface occurs. In this case the envelope can be considered as quasi-static, i.e. the average rate of mass transfer through the envelope in the radial direction is assumed to be zero. Under this condition the plasma of the stellar wind does not penetrate into the accretion lobe of the neutron star but overflows the outer edge of the envelope with the rate $\dot{M}_{\mathrm{c}}$, compressing the envelope plasma.

One faces however a slightly different situation taking into account that the "magnetic gates" during the subsonic propeller state of the neutron star are not closed completely. As has been shown above, the rate of plasma inflow into the interchange stable magnetosphere due to diffusion and the magnetic field line reconnection is $\dot{M}_{\mathrm{a}} \neq 0$. In this case the envelope remains in an equilibrium state if the amount of material inflowing from the base of the envelope to the magnetosphere is compensated by the same amount of material coming into the envelope through its outer boundary. This means that the radial drift of plasma through the envelope with the rate $\dot{M}_{\mathrm{a}}$ is expected. Hence the question about the structure of this modified envelope arises.

The characteristic time of the accretion process in the envelope can be estimated as

$t_{\mathrm{dr}}=R / V_{\mathrm{dr}}$,

\footnotetext{
${ }^{4}$ The black body radiation is assumed.
}

where $V_{\mathrm{dr}}$ is the average velocity of plasma radial drift through the envelope, which under the condition $\dot{M}_{\mathrm{a}}=$ const., can be evaluated as

$V_{\mathrm{dr}}=\frac{\dot{M}_{\mathrm{a}}}{\dot{M}_{\mathrm{c}}} V_{\mathrm{ff}}$.

On the other hand, the time scale on which the incoming energy is redistributed within the envelope (i.e. the time of envelope relaxation to the equilibrium state) is determined by the characteristic turbulence time

$t_{\mathrm{t}}=R / V_{\mathrm{t}}$.

Hence the quasi-static approximation can be applied to the modified model of the envelope if $t_{\mathrm{t}} \ll t_{\mathrm{dr}}$. Combining Eqs. (31) and (33) I find this condition to be satisfied if the spin period of the star is $P_{\mathrm{s}} \ll P_{\mathrm{qs}}$, where

$P_{\mathrm{qs}} \simeq 1.610^{3} \mu_{30}^{6 / 7} \mathrm{~m}^{-5 / 7}\left[\frac{\dot{M}_{\mathrm{a}}}{10^{13} \mathrm{~g} \mathrm{~s}^{-1}}\right]^{-1}\left[\frac{\dot{M}_{\mathrm{c}}}{10^{15} \mathrm{~g} \mathrm{~s}^{-1}}\right]^{4 / 7} \mathrm{~s}$.

This indicates that under the conditions of interest the structure of the modified envelope (with the radial plasma drift) can be considered within the quasi-static approximation and thus, the model presented by DP81 can be applied.

\section{Discussion}

The basic conclusion of the investigation presented in this paper is that the observational appearance of a neutron star in the state of subsonic propeller strongly depends on the mode by which the envelope plasma enters the magnetosphere of the star. The canonical notion that the star in this state should be observed as a non-pulsating soft X-ray source of the luminosity $L_{\mathrm{ssp}}$ is valid only in the particular case of the classical (Coulomb) diffusion. If, however, the penetration process is governed by the Bohm diffusion or/and the reconnections of the magnetic field lines, the mass accretion rate onto the star surface proves to be high enough for the accretion power to dominate the energy budget of the star. In this case the neutron star is expected to be observed as a steady low luminous $\left(L_{\mathrm{x}}=L_{\mathrm{a}, \mathrm{diff}}+L_{\mathrm{a}, \text { rec }}\right)$ accretion-powered pulsar in which most of the X-ray emission comes from the hot $\left(T_{\mathrm{cp}}\right)$ spots of the radius $\sim R_{\mathrm{cp}}$ situated in the magnetic pole regions on the surface of the neutron star (see Sect. 2.2).

The entry of the accreting plasma into the interchange stable magnetosphere of a neutron star has been investigated by Elsner \& Lamb (1984). They have shown that there are no grounds for assuming that the Bohm diffusion or the field line reconnections modes of the plasma penetrating the magnetosphere are suppressed. They evaluated the efficiency of the penetration process due to these modes to be of about $1 \%$ of the efficiency of that due to the interchange instabilities. This is too small to explain the observed luminosities of the brightest X-ray pulsars $\left(\sim 10^{37} \div 10^{38} \mathrm{erg} \mathrm{s}^{-1}\right)$ but is quite sufficient for the interpretation of the relatively low luminous X-ray sources 
$\left(L_{\mathrm{x}}<10^{36} \mathrm{erg} \mathrm{s}^{-1}\right)$. The efficiency of the plasma penetrating the magnetosphere of a neutron star evaluated by Elsner \& Lamb is comparable to that obtained in laboratory experiments (e.g. Hamasaki et al. 1974) and derived from the observations of the solar wind entering the magnetosphere of the Earth (e.g. Gosling et al. 1991). The same results were obtained in the simulations of the solar wind penetration of the Earth's magnetic field due to diffusion (Winske \& Omidi 1995) and reconnection of the field lines (Usadi et al. 1993). Thus the values $\zeta=0.1$ and $\alpha=0.1$ used in this paper seem quite reasonable. The estimation of the parameter $\lambda$ is more complicated and requires detailed study of the convective process in the envelope. This investigation, however, is beyond the scope of the present paper. Here I would like only to note that the estimate of the magnetic field scale by the size of a convection element is frequently used in the simulations of the magnetic field generation in astrophysical objects (e.g. accretion disks, active regions of the Sun, etc.).

The last point I would like to address here is the determination of the state of a low luminous neutron star by the ratio between its magnetospheric and corotation radii, $\Upsilon=R_{\mathrm{m}} / R_{\text {cor }}$. According to Eqs. (9) and (8) the value of $\Upsilon$ depends on the mass, the spin period and the dipole magnetic moment of the neutron star, and on the strength of the stellar wind in which the neutron star is situated, $\dot{M}_{\mathrm{c}}$. The parameters of the neutron star can be evaluated if it is a component of a binary system and is observed as a pulsar with the cyclotron line feature in the X-ray spectrum. In this case the value of $\Upsilon$ can be obtained combining Eqs. (2), (8) and (9):

$\Upsilon=\frac{3 \xi^{2 / 7} \mu^{4 / 7}}{L_{\mathrm{a}}^{2 / 7} R_{\mathrm{ns}}^{2 / 7}\left(G M_{\mathrm{ns}}\right)^{4 / 21} P_{\mathrm{s}}^{2 / 3}}$.

Here, $\xi=\dot{M}_{\mathrm{a}} / \dot{M}_{\mathrm{c}}$ is the efficiency of the accreting plasma penetrating the magnetic field of the star at the magnetospheric boundary.

As a rule, the value of $\xi$ is assumed to be equal to unity. This assumption is rather well justified if the neutron star undergoes disk accretion or/and if its X-ray luminosity exceeds the critical value,

$L_{\text {cr }}=3 \times 10^{36} \mu_{30}^{1 / 4} \mathrm{~m}^{1 / 2} R_{6}^{-1 / 8} \mathrm{erg} \mathrm{s}^{-1}$,

at which the cooling of the plasma over the magnetospheric boundary is effective and the boundary is interchange unstable (see Ghosh \& Lamb 1979; Arons \& Lea 1976a).
If, however, the plasma penetration into the magnetosphere of a spherically accreting neutron star is governed by the diffusion or/and the field lines reconnection processes, the assumption $\xi \simeq 1$ is not valid. In particular, in the case of subsonic propeller $\xi \lesssim 0.01$ and hence the value of $\Upsilon$ proves to be at least a factor of four smaller than that evaluated using the traditional method:

$\Upsilon(\xi)=0.27\left(\frac{\xi}{0.01}\right)^{2 / 7} \Upsilon(\xi=1)$.

This indicates that the overestimation of the parameter $\xi$ may lead to the mistaken classification of an accretionpowered source as supersonic propeller. An example of such a situation in the analysis of the low luminous state of the neutron star in the Be/X-ray transient $\mathrm{A} 0535+26$ has been recently presented by the author (Ikhsanov 2001b).

Acknowledgements. I would like to thank the anonymous referee for suggested improvements. I acknowledge the support of the Follow-up program of the Alexander von Humboldt Foundation.

\section{References}

Arons, J., \& Lea, S. M. 1976a, ApJ, 207, 914

Arons, J., \& Lea, S. M. 1976b, ApJ, 210, 792

Bondi, H., \& Hoyle, F. 1944, MNRAS, 104, 273

Davies, R. E., Fabian, A. C., \& Pringle, J. E. 1979, MNRAS, 186,779

Davies, R. E., \& Pringle, J. E. 1981, MNRAS, 196, 209

Elsner, R. F., \& Lamb, F. K. 1976, Nature, 262, 356

Elsner, R. F., \& Lamb, F. K. 1977, ApJ, 215, 897

Elsner, R. F., \& Lamb, F. K. 1984, ApJ, 278, 326

Ghosh, P., \& Lamb, F. K. 1979, ApJ, 232, 259

Gosling, J. T., Thomsen, M. F., Bame, S. J., et al. 1991, J. Geophys. Res., 96, 14097

Hamasaki, S., Davidson, R. C., Krall, N. A., \& Liewer, P. C. 1974, Nucl. Fusion, 14, 27

Ikhsanov, N. R. 2001a, A\&A, 368, L5

Ikhsanov, N. R. 2001b, A\&A, 367, 549

Ikhsanov, N. R., \& Pustil'nik, L. A. 1996, A\&A, 312, 338

Michel, F. C. 1977, ApJ, 213, 836

Priest, E. R., \& Forbes, T. G. 2000, Magnetic reconnection: MHD theory and applications (Cambridge University Press)

Shvartsman, V. F. 1970, Radiofizika, 13, 1852

Usadi, A., Kageyama, A., Watanabe, K., \& Sato, T. 1993, J. Geophys. Res., 98, 7503

Wang, Y.-M., \& Robertson, J. A. 1985, A\&A, 151, 361

Winske, D., \& Omidi, N. 1995, J. Geophys. Res., 100, 11923 